\title{
Performance advantages of CPML over UPML absorbing boundary conditions in FDTD algorithm
}

\author{
Branko D. Gvozdic, Dusan Z. Djurdjevic
}

\begin{abstract}
Implementation of absorbing boundary condition $(\mathrm{ABC})$ has a very important role in simulation performance and accuracy in finite difference time domain (FDTD) method. The perfectly matched layer (PML) is the most efficient type of ABC. The aim of this paper is to give detailed insight in and discussion of boundary conditions and hence to simplify the choice of PML used for termination of computational domain in FDTD method. In particular, we demonstrate that using the convolutional PML (CPML) has significant advantages in terms of implementation in FDTD method and reducing computer resources than using uniaxial PML (UPML). An extensive number of numerical experiments has been performed and results have shown that CPML is more efficient in electromagnetic waves absorption. Numerical code is prepared, several problems are analyzed and relative error is calculated and presented.
\end{abstract}

K e y w or d s: finite difference time domain, FDTD, perfectly matched layer, PML, convolutional PML, CPML, uniaxial PML, UPML

\section{Introduction}

With the development of technology and rapid increase of computer resources, the finite difference time domain (FDTD) method became one of the most popular numerical method in today's computational electromagnetics (CEM). FDTD method is primarily used for antenna and microwave circuits design, electromagnetic wave and radio propagation simulation and analysis, in photonics.

There is often a necessity to simulate infinite space or spatially unbounded systems in FDTD simulations. Implementation of absorbing boundary conditions (ABC) at the computational boundaries is used in infinite space FDTD simulations. One of the most important challenges in FDTD method is to efficiently and accurately implement ABCs and so to simulate the extension of the FDTD lattice to infinity.

The perfectly matched layer (PML) [1] is well known $\mathrm{ABC}$ for efficient absorption of electromagnetic waves of arbitrary polarization, angle of incidence and frequency. PML had proved efficiency for homogeneous, inhomogeneous, linear, nonlinear, dispersive and anisotropic domains. PML defined in [1] is based on non-physical field splitting of Maxwell's equations which produce a significant amount of discretization error in discrete FDTD lattice. PML with the uniaxial anisotropic medium based on electric and magnetic permittivity tensors is proposed in [2] and implemented in [3]. Uniaxial PML (UPML) [2] has the same efficiency as the split-field PML $[4,5]$, while the discretization error is decreased. After the validation of this concept [6,7], many modifications of PML were proposed $[8,9]$. Stretched coordinate (SC) formulation of Maxwell's equation extended the use of the
PML into other orthogonal coordinate systems $[10,11]$ and into general curvilinear coordinate systems [12,13], but it had weak causality. The complex frequency shifted (CFS) tensor coefficients used for PML parameters gained the causality of PML $[14,15]$.

Very effective implementation of PML based on SC, CFS and recursive convolution technique [16] is derived in [17]. Obtained convolutional PML (CPML) is entirely independent of the host medium and without the need of any modifications when applied in inhomogeneous, lossless, lossy, dispersive, nonlinear and anisotropic media, CPML is superior to the other PMLs. Improved CPMLs were recently derived in $[15,18,19]$.

In [20] comprehensive study about the choice of PML in finite difference frequency domain (FDFD) and in finite-element method (FEM), is presented.

In this paper, numerical experiments are performed to investigate absorption of electromagnetic waves and implementation of UMPL and CPML in FDTD method. In particular, 3D FDTD simulation of a differentiated Gaussian pulse propagating in free space is used for comparison of absorption for UPML and CPML ABC. Additionally, PML absorption for electromagnetic scattering from dipole antenna generating electromagnetic wave and PEC sphere is calculated in order to simulate complex wave propagation and scattering in 3D FDTD domain. Relative error for electric field is calculated for both case studies and with different thicknesses.

Implementation advantages in favor of CPML are shown in Sec. 2. Numerical results presented in Sec. 3 demonstrate that absorption characteristics of CPML are three orders of magnitude better than of UPML.

* Faculty of Technical Sciences, Kneza Milosa 7, Kosovska Mitrovica, 38220, Serbia, branko.gvozdic@pr.ac.rs 


\section{UPML and CPML Implementation in FDTD}

After Berenger's pioneering work of split-field PML [1], unsplit form with SC formulation of Maxwell's equation is proposed in [10], and independently in [11]. SC formulation enabled mapping of Maxwell's equation into complex coordinate space. Assuming that the PML parameters $s_{w}=1+\sigma_{w} / j \omega \varepsilon_{0}$ are continuous along its corresponding axis ( $w=x, y, z, \sigma_{w}$ - conductivity, $\varepsilon_{0}$ - permittivity), stretched coordinate space derivatives are defined as [12]

$$
\frac{\partial}{\partial \bar{x}}=\frac{1}{s_{x}} \frac{\partial}{\partial x}, \frac{\partial}{\partial \bar{y}}=\frac{1}{s_{y}} \frac{\partial}{\partial y}, \frac{\partial}{\partial \bar{z}}=\frac{1}{s_{z}} \frac{\partial}{\partial z} .
$$

Stretched coordinates in the complex form of Ampere's law in free space therefore are

$$
\begin{aligned}
& j \omega \varepsilon_{0} \boldsymbol{E}=\hat{x}\left(\frac{1}{s_{y}} \frac{\partial}{\partial y} \boldsymbol{H}_{z}-\frac{1}{s_{z}} \frac{\partial}{\partial z} \boldsymbol{H}_{y}\right)+ \\
& \hat{y}\left(\frac{1}{s_{z}} \frac{\partial}{\partial z} \boldsymbol{H}_{x}-\frac{1}{s_{x}} \frac{\partial}{\partial x} \boldsymbol{H}_{z}\right)+\hat{z}\left(\frac{1}{s_{x}} \frac{\partial}{\partial x} \boldsymbol{H}_{y}-\frac{1}{s_{y}} \frac{\partial}{\partial y} \boldsymbol{H}_{x}\right),
\end{aligned}
$$

and after time domain conversion

$$
\begin{aligned}
& \frac{\partial}{\partial t}\left(\varepsilon_{0} \boldsymbol{E}\right)=\hat{x}\left(\bar{s}_{y} * \frac{\partial}{\partial y} \boldsymbol{H}_{z} \bar{s}_{z} * \frac{\partial}{\partial z} \boldsymbol{H}_{y}\right)+ \\
& \hat{y}\left(\bar{s}_{z} * \frac{\partial}{\partial z} \boldsymbol{H}_{x}-\bar{s}_{x} * \frac{\partial}{\partial x} \boldsymbol{H}_{z}\right)+\hat{z}\left(\bar{s}_{x} * \frac{\partial}{\partial x} \boldsymbol{H}_{y}-\bar{s}_{y} * \frac{\partial}{\partial y} \boldsymbol{H}_{x}\right)
\end{aligned}
$$

where $*$ represents convolution as a consequence of frequency dependence of SC metrics and $\bar{s}_{w}$ is the inverse Fourier transform of $s_{w}^{-1}$.

Neither split-field PML nor SC PML are physical medium. An anisotropic, physical model composed of electric and magnetic permittivity tensors is formulated in [2] and [3] and it is referred as UPML. Thus, the general form of UPML implementation in Ampere's law in free space is

$$
j \omega \varepsilon_{0}\left(\begin{array}{ccc}
\frac{s_{y} s_{z}}{s_{x}} & 0 & 0 \\
0 & \frac{s_{x} s_{z}}{s_{y}} & 0 \\
0 & 0 & \frac{s_{x} s_{y}}{s_{z}}
\end{array}\right) \boldsymbol{E}=\nabla \times \boldsymbol{H}
$$

where $s_{x, y, z}=k_{x, y, z}+\sigma_{x, y, z} / j \omega \varepsilon_{0}$ are tensor coefficients for general media and $k_{x, y, z} \geq 1$ is real stretching coefficient contributing to an effective scaling of the mesh in the PML region. The split-field PML and UPML have the same reflection properties and propagation characteristics [4]. However, both are not efficient in absorbing evanescent waves and can cause large reflections at low frequencies due to the weak causality of PML [4,5]. A causal form of the PML is proposed and derived in [14], based on shifting the pole of $s_{w}$ into the upper-half of complex plane. Complex frequency shifted (CFS) tensor coefficients from [14] are

$$
s_{w}=k_{w}+\frac{\sigma_{w}}{\alpha_{w}+j \omega \varepsilon_{0}} .
$$

In (5) $\alpha_{w}$ is complex frequency shift parameter, with a property of homogenous conductivity.

To implement CFS in time domain, one requires Fourrier transform of $s_{w}^{-1}$

$$
\begin{aligned}
\bar{s}_{w}(t) & =F^{-1}\left(\frac{1}{k_{w}+\frac{\sigma_{w}}{\alpha_{w}+j \omega \varepsilon_{0}}}\right)= \\
& \frac{\delta(t)}{k_{w}}-\frac{\sigma_{w}}{\varepsilon_{0} k_{w}^{2}} e^{-\left(\frac{\sigma_{w}}{\varepsilon_{0} k_{w}}+\frac{\alpha_{w}}{\varepsilon_{0}}\right)} h(t)=\frac{\delta(t)}{k_{w}}+\eta_{w}(t)
\end{aligned}
$$

where $\delta(t)$ is the unit impulse function, and $h(t)$ is the unit step function. Inserting (6) into (3) yields time domain expression

$$
\begin{aligned}
& \frac{\partial}{\partial t}\left(\varepsilon_{0} \boldsymbol{E}\right)= \\
& \quad \hat{x}\left(\frac{1}{k_{y}} \frac{\partial}{\partial y} \boldsymbol{H}_{z}-\frac{1}{k_{z}} \frac{\partial}{\partial z} \boldsymbol{H}_{y}+\eta_{y} * \frac{\partial}{\partial y} \boldsymbol{H}_{z}-\eta_{z} * \frac{\partial}{\partial z} \boldsymbol{H}_{y}\right) \\
& \quad+\hat{y}\left(\frac{1}{k_{z}} \frac{\partial}{\partial z} \boldsymbol{H}_{x}-\frac{1}{k_{x}} \frac{\partial}{\partial x} \boldsymbol{H}_{z}+\eta_{z} * \frac{\partial}{\partial z} \boldsymbol{H}_{x}-\eta_{x} * \frac{\partial}{\partial x} \boldsymbol{H}_{z}\right) \\
& \quad+\hat{z}\left(\frac{1}{k_{x}} \frac{\partial}{\partial x} \boldsymbol{H}_{y}-\frac{1}{k_{y}} \frac{\partial}{\partial y} \boldsymbol{H}_{x}+\eta_{x} * \frac{\partial}{\partial x} \boldsymbol{H}_{y}-\eta_{y} * \frac{\partial}{\partial y} \boldsymbol{H}_{x}\right) .
\end{aligned}
$$

Improper implementation of convolution pairs on the right-hand side of (7) in computer algorithm leads to the usage of a huge amount of computer resources. Approaches to resolving this situation use the recursive convolution (RC) [17]. The discrete impulse response of $\eta_{w}$ and recursive convolution relation gives

$$
\psi_{w, v}(n)=b_{w} \psi_{w, v}(n-1)+c_{w} \frac{\partial}{\partial w} H_{v}(n)
$$

with

$$
\begin{gathered}
b_{w}=e^{-\left(\frac{\sigma_{w}}{\varepsilon_{0} k_{w}}+\frac{\alpha_{w}}{\varepsilon_{0}}\right) \Delta t} \\
c_{w}=\frac{\sigma_{w}}{k_{w}\left(\sigma_{w}+\alpha_{w} k_{w}\right)}\left[b_{w}-1\right] .
\end{gathered}
$$

In (9) coefficients are nonzero only in PML region and computed along with scaled tensor parameters $\sigma_{w}, \alpha_{w}$ and $k_{w}(n=i, j, k ; w=x, y, z)$. By implementing this form of $\Psi_{w, v}(n)$ good efficiency of time advancement in FDTD algorithm is achieved.

SC, CFS and RC implemented as in [17] results FDTD domain with CPML ABCs. FDTD time and space discretization of Ampere's law with CPML yields explicit update of $\boldsymbol{E}_{x}$ expressed as

$$
\begin{gathered}
\left.\boldsymbol{E}_{x}\right|_{i+\frac{1}{2}, j, k} ^{n+\frac{1}{2}}=\left.\left.C_{a}\right|_{i+\frac{1}{2}, j, k} \boldsymbol{E}_{x}\right|_{i+\frac{1}{2}, j, k} ^{n-\frac{1}{2}}+\left.C_{b}\right|_{i+\frac{1}{2}, j, k} \times \\
\left(\begin{array}{c}
\frac{\left.\boldsymbol{H}_{z}\right|_{i+\frac{1}{2}, j+\frac{1}{2}, k} ^{n}-\left.\boldsymbol{H}_{z}\right|_{i+\frac{1}{2}, j-\frac{1}{2}, k} ^{n}}{k_{y_{j}} \Delta y}-\frac{\left.\boldsymbol{H}_{y}\right|_{i+\frac{1}{2}, j, k+\frac{1}{2}} ^{n}-\left.\boldsymbol{H}_{y}\right|_{i+\frac{1}{2}, j, k-\frac{1}{2}} ^{n}}{k_{z_{k}} \Delta z} \\
\left.\psi_{\boldsymbol{E}_{x, y}}\right|_{i+\frac{1}{2}, j, k} ^{n}-\psi_{\left.\boldsymbol{E}_{x, z}\right|_{i+\frac{1}{2}, j, k} ^{n}}^{n}
\end{array}\right)
\end{gathered}
$$


In $(10) \psi_{E x, y}, \psi_{E x, z}$ are PML coefficients existing only in PML region, updated as follows

$$
\begin{aligned}
\left.\psi_{\boldsymbol{E}_{x, y}}\right|_{i+\frac{1}{2}, j, k} ^{n} & =\left.b_{y_{j}} \psi_{\boldsymbol{E}_{x, y}}\right|_{i+\frac{1}{2}, j, k} ^{n-1} \\
& +c_{y_{j}}\left(\frac{\left.\boldsymbol{H}_{z}\right|_{i+\frac{1}{2}, j+\frac{1}{2}, k} ^{n}-\left.\boldsymbol{H}_{z}\right|_{i+\frac{1}{2}, j-\frac{1}{2}, k} ^{n}}{\Delta y}\right),
\end{aligned}
$$

$$
\begin{aligned}
\left.\psi_{\mathbf{E}_{x, z}}\right|_{i+\frac{1}{2}, j, k} ^{n} & =\left.b_{z_{k}} \psi_{\mathbf{E}_{x, z}}\right|_{i+\frac{1}{2}, j, k} ^{n-1}+ \\
& c_{z_{k}}\left(\frac{\left.\boldsymbol{H}_{y}\right|_{i+\frac{1}{2}, j, k+\frac{1}{2}} ^{n}-\left.\boldsymbol{H}_{y}\right|_{i+\frac{1}{2}, j, k-\frac{1}{2}} ^{n}}{\Delta z}\right) .
\end{aligned}
$$

Coefficients $C_{a}$ and $C_{b}$ are used for update of $\boldsymbol{E}_{x}$ field and they are calculated as

$$
\begin{aligned}
\left.C_{a}\right|_{i+\frac{1}{2}, j, k} & =\frac{1-\frac{\sigma_{i+\frac{1}{2}, j, k} \Delta t}{2 \varepsilon_{i+\frac{1}{2}, j, k}}}{1+\frac{\sigma_{i+\frac{1}{2}, j, k} \Delta t}{2 \varepsilon_{i+\frac{1}{2}, j, k}}}, \\
\left.C_{b}\right|_{i+\frac{1}{2}, j, k}= & \frac{\frac{\Delta t}{\varepsilon_{i+\frac{1}{2}, j, k}}}{1+\frac{\sigma_{i+\frac{1}{2}, j, k} \Delta t}{2 \varepsilon_{i+\frac{1}{2}, j, k}}} .
\end{aligned}
$$

Similar expressions are derived for five remaining field components $\left(E_{y}, E_{z}, H_{x}, H_{y}\right.$ and $\left.H_{z}\right)$ for 3D FDTD domain, with the adequate replacement of $(i, j, k)$ and $(x, y, z)$.

Efficiency of CPML is mainly dependent on the proper choice of parameters. Parameters can be spatially graded in different ways, but two the most successful are polynomial and geometric grading. In this paper, the polynomial grading is used. PML parameters are scaled as follows $[3,21]$

$$
\begin{aligned}
k_{w}(l) & =1+\left(k_{w, \max }-1\right)\left(\frac{l}{d}\right)^{m}, \\
\sigma_{w}(l) & =\sigma_{w, \max }\left(\frac{l}{d}\right)^{m}, \\
\alpha_{w}(l) & =\alpha_{w, \max }\left(\frac{d-l}{d}\right)^{m_{a}}, \quad 0 \leq l \leq d
\end{aligned}
$$

where $l$ is PML loss depth, $d$ is PML thickness, $m$ and $m_{a}$ are the scaling orders. Conductivity $\sigma_{w}$ is scaled to be 0 at the PML surface $(l=0)$ and $\sigma_{w, \max }$ at the PM outer boundary $(l=d)$. Stretching coefficient $k_{w}$ is 1 at the beginning of PML and $k_{w \text {,max }}$ at the end of PML. Complex frequency shift parameter $\alpha_{w}$ has a maximum at the front of PML, thereby decreasing reflection error of evanescent modes. Inside the PML, $\alpha_{w}$ is decreased to a minimum in order to appropriately decay low frequencies of the wave propagating [17].

The proper choice of PML parameters is decisive for PML efficiency. Trade-off between reflection error from the PML outer boundary and discretization error from the front PML interface have to be properly balanced.
If $\sigma_{w, \max }$ is too small, reflection error from the back of PML is dominant, while for large $\sigma_{w, \max }$ discretization error is significantly increased. In [17] optimal choice for polynomial graded $\sigma_{w, \max }$ is proposed, derived for general media as

$$
\sigma_{w, \mathrm{opt}}=\frac{0.8(m+1)}{Z_{0} \Delta_{w} \sqrt{\varepsilon_{r, \mathrm{eff}}} \mu_{\mathrm{r}, \mathrm{eff}}},
$$

where $Z_{0}$ is impedance of free space, $\Delta_{w}$ is spatial step in $w=x, y, z$ direction, $\varepsilon_{r, \text { eff }}$ and $\mu_{r \text { eff }}$ are effective relative permittivity and permeability, respectively.

Optimal CPML parameters are [8,17]: $0.75 \sigma_{w, \text { opt }}<$ $\sigma_{w, \max }<1.4 \sigma_{w, \text { opt }}, 7<k_{w, \max }<20$, and $0.15<\alpha_{w}<$ 0.3 . Scaling orders are in ranges: $3 \leq m \leq 4$, and $m_{a} \approx$ 1 .

It can be seen that CPML is simpler to implement resulting also in more storage-efficient algorithm than UPML implementation. In particular, UPML is quite simple to implement in existing FDTD codes, but with the cost of doubling memory requirements through entire FDTD domain. Usage of triple-nested loops for the fields inside the computational domain, and individual loops in UPML region saves the memory, but it increases the complexity of programming.

In the case of CPML implementation in FDTD, CPML variables are stored only in PML region, hence the superior memory efficiency over the UPML. Furthermore, CPML implementation remains unchanged in the case of homogeneous, inhomogeneous, lossy and dispersive medium. On the contrary, UPML requires additional two variables per field component in all those mediums.

In order to estimate the advantages of CPML over the UPML in FDTD, simulations are performed and numerical results are obtained for two different electromagnetic problems. The first problem is 3D FDTD simulation of electromagnetic wave propagation in free space with a differentiated Gaussian pulse as a source. The second problem is 3D FDTD electromagnetic scattering from PEC sphere and dipole antenna centered in the computational domain as an example of complex FDTD case. For both cases, relative error at two probe points is calculated comparing absorption for two PMLs.

The explicit FDTD algorithm is used and calculated with original $\mathrm{C}++$ codes. Numerical results of the electromagnetic field and relative error graphs are plotted with the command-line driven Gnuplot graphing utility.

\section{Numerical Results and Discussions}

\section{D Simulation of Gaussian Pulse in Free Space}

Propagation of differentiated Gaussian pulse in free space in 3 D FDTD domain is simulated in $200 \times 200 \times 200$ space lattice, with 1-mm-square cells and time-step of $d t=1.906575$ ps (0.99 times of Courant limit). Duration of simulation is 600 time-steps (1.143945 ns). Gaussian 


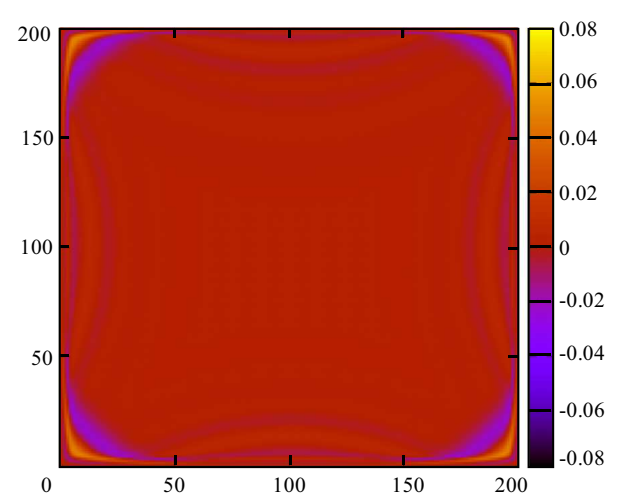

Fig. 1. $E_{z}$ field component for 10 cell-thick UPML after 300 timesteps, $x y$ plane

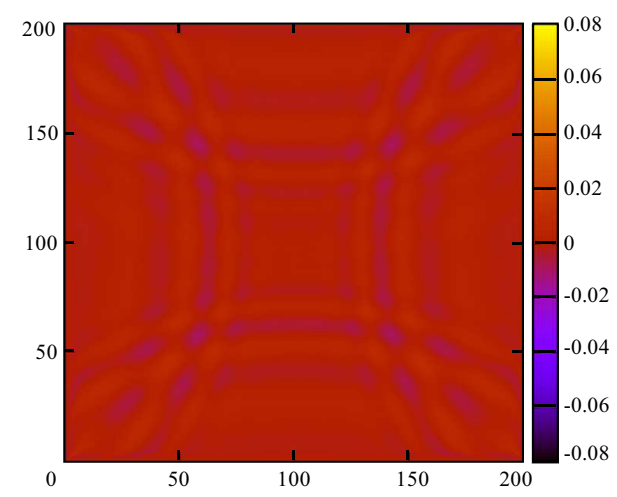

Fig. 3. $E_{z}$ field component for 10 cell-thick UPML after 400 timesteps, $x y$ plane

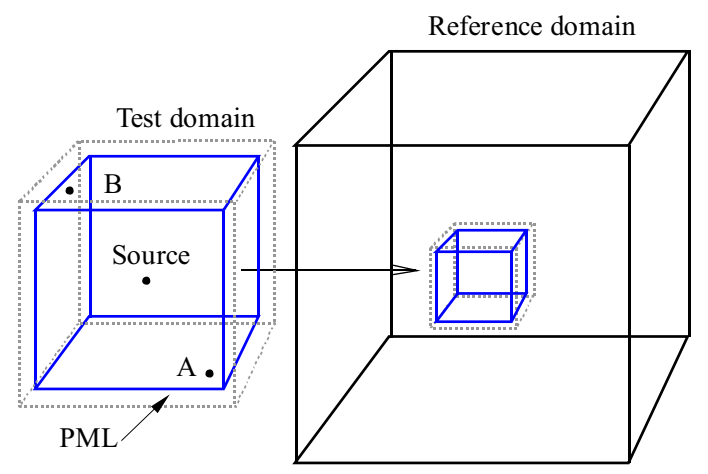

Fig. 5. Illustration of test and reference FDTD domain for relative error calculation for Gaussian pulse

pulse is placed in the center of the computational domain with time function

$$
J_{(x, y, z, t)}=-2\left[\left(t-t_{d}\right) / g_{w}\right] e^{-\left[\left(t-t_{d}\right) / g_{w}\right]^{2}},
$$

where $g_{w}=30 \mathrm{ps}$ is half-width of Gaussian pulse and $t_{d}=4 g_{w}$ is a time delay. For comparison purposes, FDTD domain is terminated with 10-cell thick UPML and CPML, with polynomial grading defined in (14), (15) and (16).

Numerical results shown in Fig. 1 present $E_{z}$ field distribution for 10-cell thick UPML after 300 time steps, over the xy plane, with $m=3, \sigma_{w, \max }=0.75 \sigma_{w, \text { opt }}$

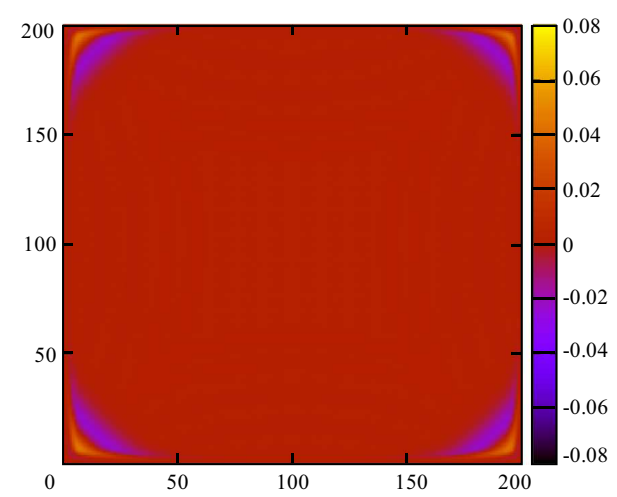

Fig. 2. $E_{z}$ field component for 10 cell-thick CPML after 300 timesteps, $x y$ plane

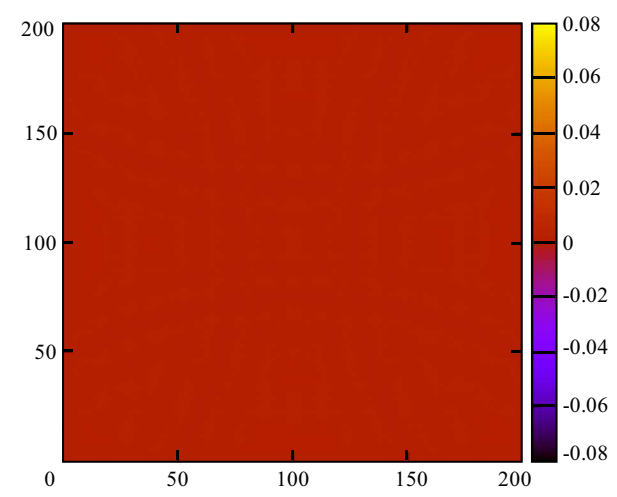

Fig. 4. $E_{z}$ field component for 10 cell-thick CPML after 400 timesteps, $x y$ plane

(with $\sigma_{w, \text { opt }}$ from (17)), $k_{w, \max }=15$ and $\alpha_{w}=0$, yielding the properties of UPML. Figure 2 shows the $E_{z}$ field distribution for 10-cell thick CPML after 300 time steps, over the $x y$ plane, with the same PML parameters except for $\alpha_{w}=0.24$ and $m_{a}=1$, yielding the properties of CPML. A significant amount of numerical dispersion reflecting from the computational domain outer boundary can be seen in Fig. 1, in comparison with results shown in Fig. 2. Numerical results presented in Fig. 2 show fine absorption of $E_{z}$ field component, without reflecting any field components back to the computational domain.

Numerical results shown in Fig. 3 and Fig. 4 present $E_{z}$ field distribution for 10-cell thick UPML and CPML after 400 time steps, over the $x y$ plane, respectively. The increase of numerical dispersion of $E_{z}$ field component, reflecting back from UPML into the computational domain, can be seen in Fig. 3. Such numerical artifacts are intolerable in simulations where the precise calculation is required. Consequently, efficient CPML absorption of electromagnetic wave can be seen in Fig. 4 (steady-state).

In order to demonstrate benefits of CPML over UPML $\mathrm{ABCs}$, the relative error is calculated for electric field $E$ at points $\mathrm{A}$ and $\mathrm{B}$, as shown in Fig. 5. Test domain with $40 \times 40 \times 40$ cell grid and reference domain with $400 \times$ $400 \times 400$ cell grid are used for relative error calculation, 


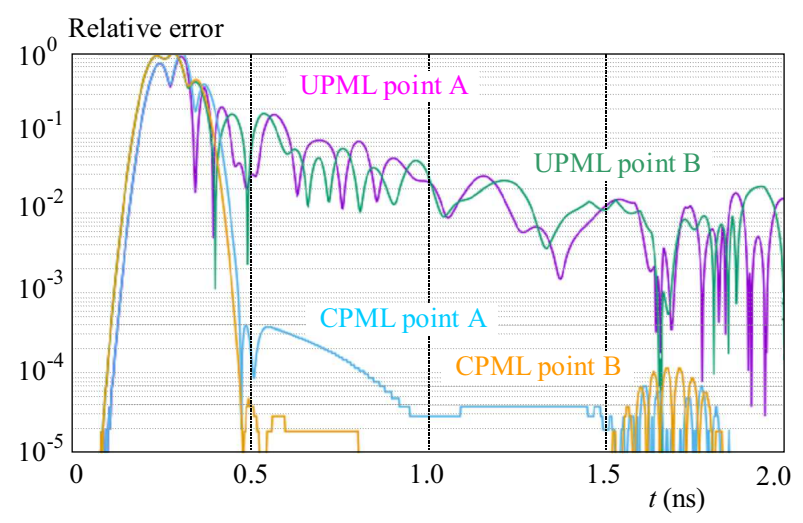

Fig. 6. Relative error for 10 cell-thick PMLs with Gaussian pulse

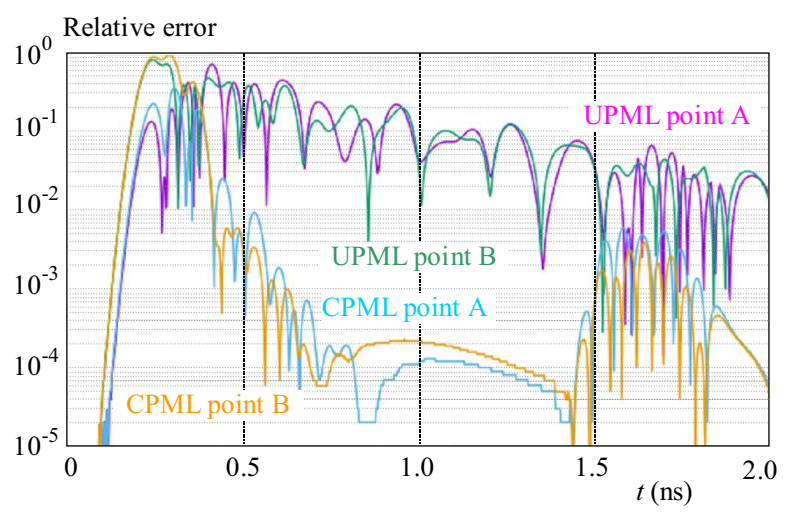

Fig. 7. Relative error for 5 cell-thick PMLs with Gaussian pulse

with:

$$
\left.R\right|_{i, j, k} ^{n}=\frac{|\boldsymbol{E}| \begin{array}{l}
n \\
i, j, k
\end{array}-\boldsymbol{E}_{\mathrm{ref}}\left|\begin{array}{l}
n \\
i, j, k
\end{array}\right|}{\left|\boldsymbol{E}_{\text {ref } \max }\right|_{i, j, k} \mid} .
$$

In (19), $\left.E\right|_{i, j, k} ^{n}$ is electric field at probe point and time-step $n$ in test domain, $\left.E_{\text {ref }}\right|_{i, j, k} ^{n}$ is electric field at probe point and time-step $n$ in reference domain and $\left.E_{\text {ref } \max }\right|_{i, j, k} ^{n}$ is the maximum amplitude of the reference field at probe point over the time-stepping range of interest. Reference domain is kept sufficiently large to avoid reflection from the walls of FDTD domain during 1000 time-steps of interest. The same source function as for Gaussian pulse propagation in free space is used, with $g_{w}=50 \mathrm{ps}, t_{d}=4 g_{w}$, in test and reference domain. Identical source location (centered in FDTD grid) is used for both domains and probe points are at the same position relative to the source. Point A $(2,2,2)$ and point $\mathrm{B}(38,20,38)$ in test domain correspond to point A $(182,182,182)$ and point B $(218,200,218)$ in reference domain. Relative error for 10-cell thick and 5-cell thick different PMLs are obtained, with the same parameters like in the case for numerical results.

The relative error for the calculated $E$ field at two probe points for 10-cell thick UPML and CPML is plotted in Fig. 6. Comparing UPML and CPML graphs it is clearly visible that CPML provides error reduction for more than three orders of magnitude on a logarithmic scale. Late time reflection error with very slow decay can be seen on UMPL graphs as a consequence of lowfrequency evanescent fields interaction with PML layers.

Relative error for calculated $E$ field at points A and $\mathrm{B}$ in the case of 5-cell thick UPML and CPML is shown in Fig. 7. Observing the CPML graphs, the early time error peaks are due to discretization error, which slowly decay after time-stepping increase. Nevertheless, it is shown that, compared with UPML, even the 5-cell thick CPML exhibits three orders of magnitude of error reduction.

\section{$3 D$ simulation of dipole antenna with PEC sphere}

The electromagnetic wave scattering from PEC sphere in $3 \mathrm{D}$ FDTD domain is simulated in $300 \times 300 \times 300$ space lattice, with 1-mm-square cells and time-step of $d t=1.906575 \mathrm{ps}$ (0.99 times of Courant limit). The source of the electromagnetic wave is dipole antenna placed in the center of FDTD computational domain. PEC sphere is made from aluminum and it is placed like in Fig. 12. Simulation time was 1000 time-steps. The $z$-directed dipole antenna is driven with differentiated Gaussian pulse as source function with a time signature of (15) and $g_{w}=30 \mathrm{ps}, t_{d}=4 g_{w}$. FDTD domain is surrounded with 10-cell thick PML ABCs with polynomial grading defined in (14), (15) and (16).

Numerical results presented in Fig. 8 show $E_{z}$ field component over the $x y$ plane, at $500^{\text {th }}$ time-step for 10 cell thick UPML, with $m=3, \sigma_{w, \max }=0.75 \sigma_{w, \mathrm{opt}}$ (with $\sigma_{w, \text { opt }}$ from (17)), $k_{w, \max }=15$ and $\alpha_{w}=0$, yielding the properties of UPML. $E_{z}$ field after 500 time steps for 10cell thick CPML, over the $x y$ plane, with the same PML parameters except for $\alpha_{w}=0.24$ and $m_{a}=1$, yielding the properties of CPML, is shown in Fig. 9. Results presented in Fig. 8 show that UPML layers reflect incident field components as well as scattering field components from PEC sphere, hence completely undermining the interpretation of the numerical results. Results presented in Fig. 9 contrary show that CPML layers linearly absorb all impinging field components.

$E_{z}$ field component is given in Fig. 10 in the case of UPML ABCs, over the $x y$ plane, close to the end of the simulation, after 700 time-steps. The plot clearly indicates that the reflected field components are propagated back to the dipole, centered in the computational domain, confronting the late time incident field components from the source. In Fig. 11, it is shown that after 700 time-steps in the case of CPML, only late time incident field components are visible close to the dipole, slowly approaching to steady state. Results indicate that CPML permits much smaller FDTD space lattice to be employed while retaining accuracy.

Relative error in the case of electromagnetic scattering with dipole and sphere is calculated as well, by using the relation (19). Test domain with dimensions: $40 \times 40 \times 40$ cells and reference domain with $400 \times 400 \times 400$ FDTD lattice are used. Probe point $\mathrm{A}$ is at the same position as in the previous example, while point $\mathrm{B}$ is at $(38,2,20)$ in 


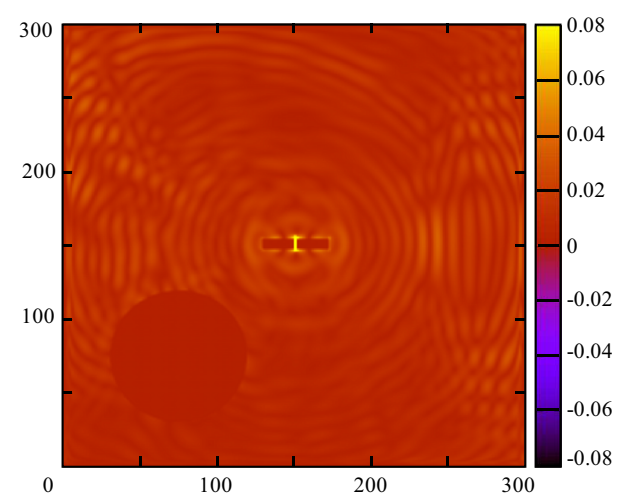

Fig. 8. $E_{z}$ field component for 10 cell-thick UPML after 500 timesteps, $x y$ plane

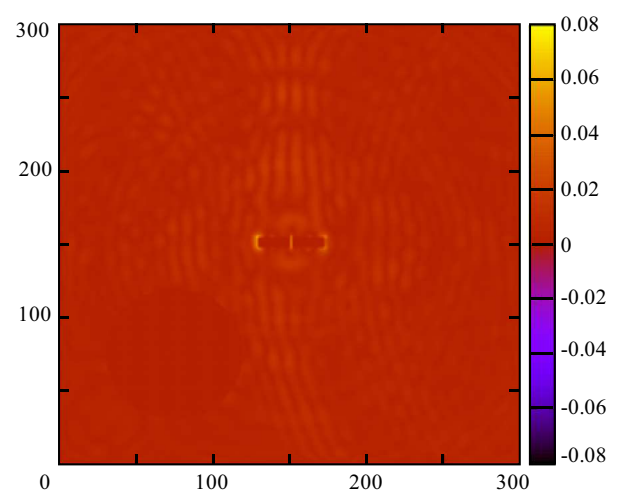

Fig. 10. $E_{z}$ field component for 10 cell-thick UPML after 700 time-steps, $x y$ plan

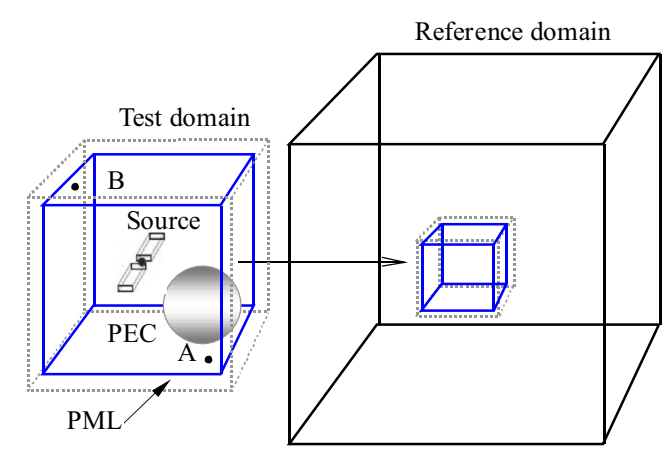

Fig. 12. Illustration of test and reference FDTD domain for relative error calculation for dipole and sphere

a test domain and $(218,182,200)$ in reference domain, as illustrated in Fig. 12.

Simulation parameters, source parameters and UPML and CPML parameters are the same as for dipole and sphere numerical results. The error is calculated for 10cell thick PMLs and shown in Fig. 13. Two CPML graphs on Fig. 13 illustrate superior absorption properties of CPML over the UPML in complex FDTD case.

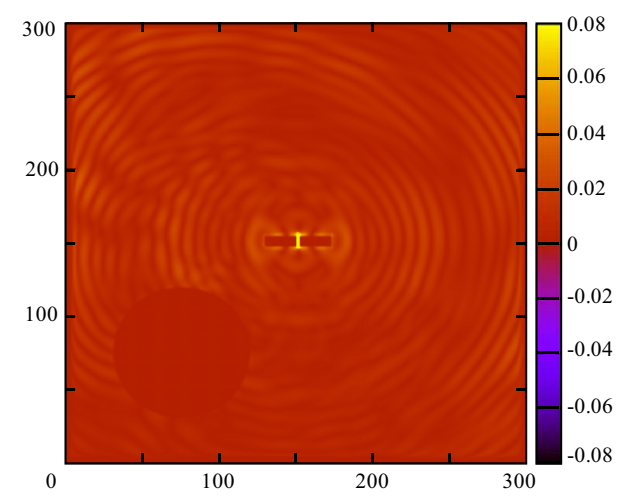

Fig. 9. $E_{z}$ field component for 10 cell-thick CPML after 500 timesteps, $x y$ plane

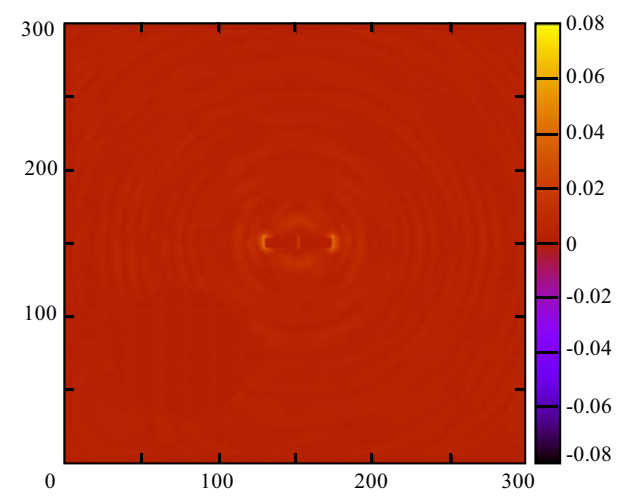

Fig. 11. $E_{z}$ field component for 10 cell-thick CPML after 700 time-steps, $x y$ plane

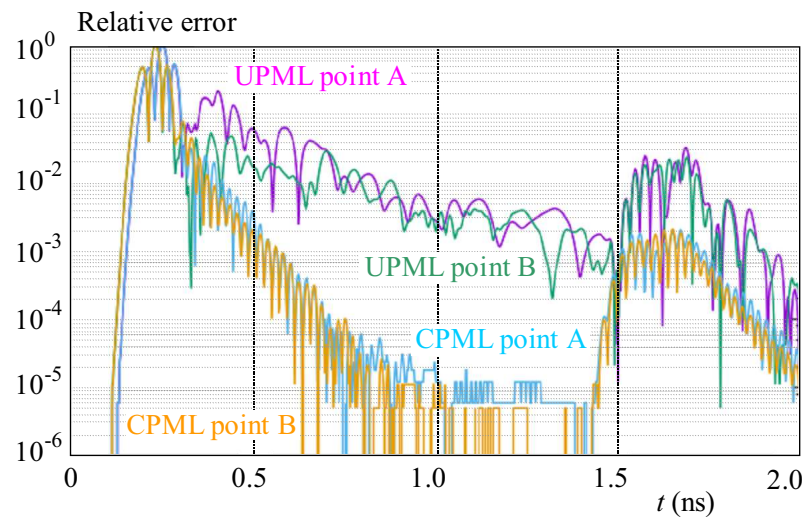

Fig. 13. Relative error for 10 cell-thick PMLs with dipole and sphere

\section{Conclusions}

In this paper, we compared two main algorithms of PML ABC used today in FDTD method: UPML and CPML. Basic theory and algorithm steps concerning UPML and CPML in FDTD method are briefly explained and discussed. Extensive numerical FDTD simulations were performed by using the original computer 
code. Presented numerical results clearly demonstrate advantages of CPML absorption of electromagnetic waves over UPML in FDTD algorithm. The main advantages of CPML boundary conditions are highlighted: they are much simpler to implement in FDTD code and more computationally efficient than UPML.

\section{REFERENCES}

[1] J. P. Berenger, "A Perfectly Matched Layer for the Absorption of Electromagnetic Waves", Journal of Computational Physics, vol.114, pp.185-200, 1994.

[2] Z. S. Sacks, D. M. Kingsland, R. Lee and J. F. Lee, "A Perfectly Matched Anisotropic Absorber for Use as an Absorbing Boundary Condition", IEEE Trans. Antennas Propagat., vol. 43, 1460-1463, 1995.

[3] S. D. Gedney, "An Anisotropic Perfectly Matched Layer Absorbing Media for the Truncation of FDTD Lattices", IEEE Trans. Antennas Propagat., vol. 44, 1630-1639, 1996.

[4] J. P. Berenger, "Numerical Reflection from FDTD PMLs: A Comparison of the Split PML with the Unsplit and CFS PMLs", IEEE Trans. Antennas Propagat., vol. 50, 258-265, 2002.

[5] D. Correia and J. M. Jin, "Performance of Regular PML, CFS-PML, and Second-Order PML for Waveguide Problems", Microwave and Optical Technology Letters, vol. 4\$ 2121-2126, 2006.

[6] D. S. Katz, E. T. Thiele and A. Taflove, "Validation and Extension to Three-Dimensions of the Berenger PML Absorbing Boundary Condition for FDTD Meshes", IEEE Microwave Guided Wave Lett., vol. 4, 268-270, 1994.

[7] J. Demoerloose and M. A. Stuchley, "Reflection Analysis of PML ABC's for Low-Frequency Applications", IEEE Microwave Gui -ded Wave Lett., vol. 6, 177-179, 1996.

[8] J. P. Berenger, "Improved PML for the FDTD Solution of Wave-Structure Interaction Problems", IEEE Trans. Antennas Propagat., vol. 45, 466-473, 1997.

[9] D. M. Sullivan, "A Simplified PML for Use with the FDTD Method", IEEE Microwave Guided Wave Lett., vol. 6, 97-99, 1996.

[10] W. C. Chew and W. H. Weedon, "A 3D Perfectly Matched Medium from Modified Maxwell's Equations with Stretched Coordinates", IEEE Microwave Guided Wave Lett., vol. 7, 599-604, 1994.

[11] C. M. Rappaport, "Perfectly Matched Absorbing Boundary Conditions based on Anisotropic Lossy Mapping of Space", IEEE Microwave Guided Wave Lett., vol. 5, 90-92, 1995.

[12] F. L. Teixeira and w. C. Chew, "PML-FDTD in Cylindrical and Spherical Coordinates", IEEE Microwave Guided Wave Lett., vol. 7, 285-287, 1997.

[13] F. L. Teixeira, K. P. Hwang, W. C. Chew and J. M. Jin, "Conformal PML-FDTD Schemes for Electromagnetic Field Simulations: A Dynamic Stability Study", IEEE Trans. Antennas Propagat., vol. 49, 902-907, 2001.
14] M. Kuzuoglu and, R. Mitra, "Frequency Dependence of the Constitutive Parameters of Causal Perfectly Matched Anisotropic Absorbers", IEEE Microwave Guided Wave Lett., vol. 6, 447-449, 1996

15] J. P. Berenger, "An Optimized CFS-PML for Wave-Structure Interaction Problems", IEEE Transactions on Electromagnetic Compatibility, vol. 54, 351-358, 2012.

[16] R. J. Luebbers and F. Hunsberger, "FDTD for Nth-Order Dispersive Media", IEEE Trans. Antennas Propagat., vol. 40, 1297-1301, 1992.

[17] J. A. Roden and S. D. Gedney, " Convolutional PML (CPML): An Efficient FDTD Implementation of the CFS-PML for Arbitrary Media", Microwave Optical Tech. Lett., vol. 27, 334-339, 2000

[18] I. Giannakis and A. Giannopoulos, "Time-Synchronized Convolutional Perfectly Matched Layer for Improved Absorbing Performance in FDTD", IEEE Antennas and Wireless Propagation Letters, vol. 14, 690-693, 2015

[19] Z. H. Li and Q. H. Huang, "Application of the Complex Frequency Shifed Perfectly Matched Layer Absorbing Boundary Conditions in Transient Electromagnetic Method Modelling", Chinese J. Geophys, vol. 57, 1292-1299, 2014.

[20] W. Shin and S. Fan, "Choice of the Perfectly Matched Layer Boundary Condition for Frequency-Domain Maxwell's Equations Solvers", Journal of Computational Physics, vol. 231.8, 3406-3431, 2012.

21] A. Taflove and S. C. Hagness,, Computational electrodynamics: The Finite-Difference Time-Domain Method, 3rd ed., Norwood, USA, Artech House, 2005.

Received 29 November 2016

Branko Gvozdic was born in Kosovska Mitrovica, Serbia, in 1985. He received BSc and MSc degrees in electrical and computer engineering, in 2008 and 2010, from the Faculty of Technical Sciences in Kosovska Mitrovica, University of Pristina, Serbia. He is $\mathrm{PhD}$ candidate in the Faculty of Technical Sciences in Kosovska Mitrovica, University of Pristina, Serbia. In 2012 he became a teaching assistant at the Faculty of Technical Sciences at the University of Pristina. Areas of research include finite difference time domain method, modeling of electromagnetics, photonics design.

Dusan Djurdjevic was born in Pristina, Serbia, in 1960. He received $\mathrm{BE}$ degree in electrical engineering from the University of Pristina in 1983, MS degree from the University of Nis in 1992, and PhD degree (Doctorate in Technical Sciences) from the University of Belgrade in 1996. From 2000 to 2004 he joined the School of Electrical Engineering at the Nottingham University, United Kingdom, as a research associate. In 2004 and 2005 he joined the Dept. of Electrical and Computer Engineering at the University of Massachusetts, Dartmouth, USA, as a visiting scholar. In 1997 he became a Lecturer and in 2011 associate professor, teaching Electromagnetics, Theory of electric circuits, Antennas and propagations and Microwaves. His research interests include analytical and numerical modeling of electromagnetics in antenna and photonics design. 